use of $t$-butoxycarbonyl-substituted hydrazones (rather than $\mathrm{N}-\mathrm{H}$ hydrazones) in conjunction with an acidic catalyst allows a 'traceless' carbon-carbon bondforming process - directly analogous to the original result of Stevens - to proceed at temperatures as low as $125^{\circ} \mathrm{C}(\mathbf{7} \rightarrow \mathbf{8}$, Fig. 2). These changes certainly bring the conditions into the realm of utility for application in non-trivial contexts.

Aromatic, heteroaromatic and aliphatic aldehydes are all competent substrates for the two-step process of hydrazide formation followed by thermolytic carbon-carbon bond formation (Fig. 2). This reaction offers a new method to convert readily available benzaldehydes and heteroaromatic aldehydes into homoallylated arenes (see $\mathbf{9} \rightarrow \mathbf{1 0}$, for example). In the context of aliphatic aldehydes, the convergent union of two enantioenriched substrates to generate products with a 1,6-diastereorelationship (such as in the conversion of $\mathbf{1 1}$ to $\mathbf{1 2}$ ) is powerful for synthesis. Hydrazides derived from $\alpha$-chiral aldehydes do not epimerize to a significant extent under the rearrangement conditions, and the configuration of the allylic, hydrazidebearing stereogenic centre in the intermediate does not need to be controlled for productive reactivity. Both of these features engender potential utility for complex-molecule synthesis.

Although the reaction is traceless in the sense that the aldehyde and the N-Boc hydrazide functional groups are lost in the course of the thermolytic rearrangement (as dinitrogen, isobutylene and carbon dioxide), there remains a retron for application of this method. Overall, the process can be considered as the formation of a homoallylic carbon-carbon bond; therefore, in many cases when one is confronted with the need to unite fragments of a target molecule that are joined by an $E$-alkene, this reaction has the potential to be useful. Its usefulness will, of course, depend on the ready introduction of the precursor aldehyde and $\mathrm{N}$-Boc hydrazide into the starting materials of choice. This new method provides a new means of connecting building blocks to form products that would normally be made using olefination methods. It is also a potentially valuable alternative to Suzuki-type reactions, which can be used to forge the allylic carboncarbon bond.

Clearly, this work is a step in the right direction towards a new carbon-carbon bond construction of broad application. At this point, the moderately high temperatures required in conjunction with the very strong Brønsted acid catalyst triflimide preclude the use of acid-labile groups, including a large repertoire of the protective groups frequently used in natural-product synthesis. Furthermore, the yields are typically in the $50-75 \%$ range. Both of these limitations might be addressed by continued refinement of hydrazone substrates and reaction conditions that could lead to lower temperatures. Ultimately, although the reaction originally described by Stevens had been considered a curiosity and largely ignored, it might eventually find itself on the synthetic chemist's list of 'go-to methods' for convergent synthesis of complex molecules.

Sarah E. Steinhardt and Christopher D. Vanderwal are in the Department of Chemistry, University of California, Irvine, California 92697-2025, USA. e-mail: cdv@uci.edu

References

1. Stevens, R. V., McEntire, E. E., Barnett, W. E. \& Wenkert, E. J. Chem. Soc. Chem. Commun. 662-663 (1973).

2. Mundal, D. A., Avetta, C. T. Jr \& Thomson, R. J. Nature Chem. 2, 294-297 (2010)

3. Corey, E. J. \& Cheng, X.-M. The Logic of Chemical Synthesis (Wiley, 1989)

4. Mundal, D. A., Lee, J. J. \& Thomson, R. J. J. Am. Chem. Soc. 130, 1148-1149 (2008).

Mundal, D. A., Lutz, K. E. \& Thomson, R. J. Org. Lett. 11, 465-468 (2009).

\title{
SUPERCOOLED WATER
}

\section{Ice ice maybe}
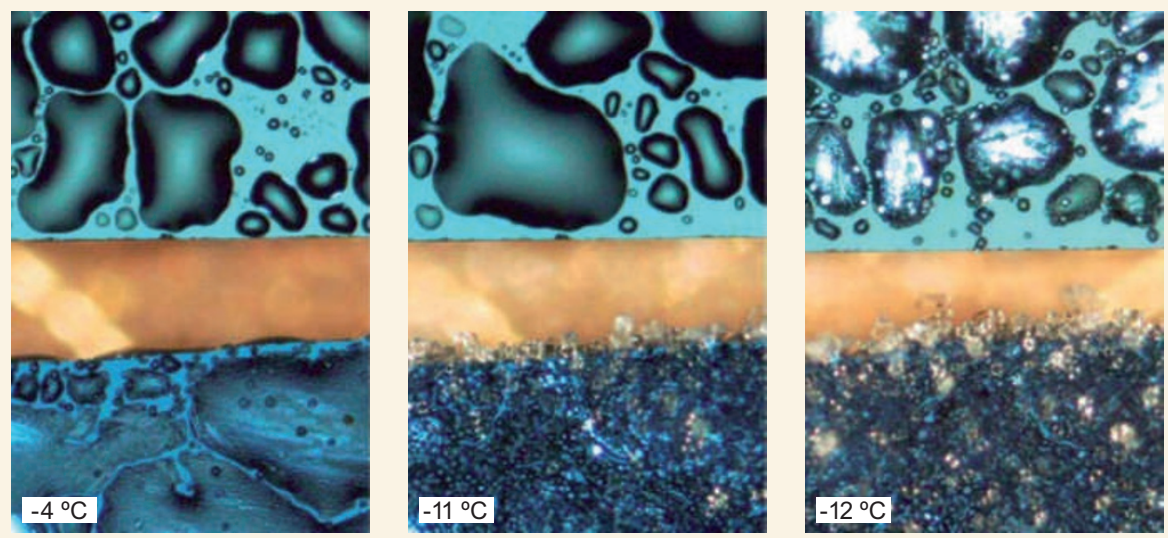

Supercooled water can remain in the liquid state at temperatures as low as $-40^{\circ} \mathrm{C}$ - providing no seeding nuclei are available - but it has long been known that electric fields near charged surfaces can influence water's freezing temperature. It has so far, however, proved difficult to study the effect of the electric field alone, because nucleation is also induced by conducting surfaces. Now Igor Lubomirsky and colleagues at the Weizmann Institute of Science, Israel, have used pyroelectric materials - polar insulators that create a transitory electric field when heated or cooled - to do just that (Science 327, 672-675; 2010).

Pyroelectric materials create equal but opposite charges at their opposing surfaces, so Lubomirsky and colleagues could use the same compound, $\mathrm{LiTaO}_{3}$, to study the effects of both positive and negative charges on the freezing of supercooled water. The pyroelectric samples were exposed to a humid atmosphere and cooled until water droplets condensed on their surfaces and later froze. They found that supercooled water froze at $-12.5^{\circ} \mathrm{C}$ on an uncharged surface, $-7^{\circ} \mathrm{C}$ on a positively charged surface and $-18{ }^{\circ} \mathrm{C}$ at a negatively charged surface.

Similar results were observed when experiments were repeated using $\mathrm{SrTiO}_{3}$. Water droplets froze at $-4^{\circ} \mathrm{C}$ on positively charged pyroelectric quasi-amorphous films (pictured, bottom left) and at $-12{ }^{\circ} \mathrm{C}$ on non-pyroelectric amorphous films (pictured, top right).

\section{GAVIN ARMSTRONG}

The original version of this story first appeared on the Research Highlights section of the Nature Chemistry website. 